\title{
NOCTURNAL MYOCLONUS
}

BY

\author{
CHARLES P. SYMONDS
}

From Guy's Hospital, London

It is not at all uncommon to find people generally considered to be in normal health, and without any clinical symptoms of epilepsy, who say that occasionally when just dropping off to sleep they experience a sudden, involuntary jerk of the limbs or body. This is momentary and as a rule confined to the lower limbs. The subject wakes with a start, and usually then drops off to sleep, but the jerk may sometimes recur. It never occurs under other conditions, happening, therefore, in bed, or much less often, on dozing during the day. It is of comparatively rare occurrence in persons who have the liability and does not appear to be precipitated by fatigue or anxiety. For convenience in discussion I shall refer to this phenomenon as nocturnal jerks, to be distinguished from nocturnal myoclonus. For many years I have suspected that the liability to nocturnal jerks, if excessive, may be associated sooner or later with the manifestation of epilepsy. This suspicion has been supported by routine enquiry for a past history of the nocturnal jerks in patients presenting with epilepsy in later years. In a few such cases it has been possible to discover that a similar excessive liability to the jerks has been present in near relatives, though they have never had any epileptic attacks.

There is another group of patients who suffer from involuntary clonic movements at night which are greatly in excess of those which may occur in normal persons. For this condition I propose the term nocturnal myoclonus and shall relate some examples.

\section{Case Reports}

Case 1.-A successful and active man of business. aged 57, presented himself in 1951 with the complaint of involuntary movements preventing sleep. There was no family history of epilepsy. In the first world war he sustained a gun-shot wound of the head, involving the scalp only. There was brief retrograde amnesia and a post-traumatic amnesia of about 48 hours, with no sequelae. In 1933 , at the age of 39 , he began to have occasional involuntary jumps of the trunk and limbs at the moment of going off to sleep. At first there would be a single jerk only, but as time went on there would be repetition at intervals of a minute for an hour or two. He stated that he could always tell if he were going to have the jumps because they were preceded by hypnogogic hallucinations as soon as he closed his eyes. He did not have the jumps every night but for a series of nights, after which there would be an interval of freedom. In the early stages of his complaint this had been as long as a year, but now freedom for six weeks was exceptional,, 0 and the series of bad nights lasted two or three weeks. In the past two years in association with each jump he had had a curious feeling in his head, which he likenedबi to the sensation experienced when his knee jerk was elicited, as if, he said, he had been hit on the head and this caused the jerk of his whole body. He suffered from flatulent dyspepsia and thought he was more apt to have the jerks when this was bad. He had been discovered seven years previously to have a high blood pressure, and this was $200 / 130 \mathrm{~mm} . \mathrm{Hg}$ at the time of examination. Otherwise he was in good health without abnormal physical signs. His only complaint was of loss of sleep when disturbed by the jumps.

No E.E.G. was obtained in this case. Radiographs of the skull were normal.

The possible significance of the head injury in this case cannot be disregarded. The same question will arise in Case 3.

Case 2.-A business man, aged 56, was seen in 1951, complaining of involuntary movements at night interfering with sleep. There was no relevant family or past history. At the age of $\mathbf{4 0}$ he began to have occasional involuntary jerks in bed at night to which he did not pay much attention. At the age of 46 there was an episode which was at the time alarming. He had been working after dinner and because he was feeling strained and tense took a stroll in the garden. Suddenly he "felt he was going" and collapsed in a heap with a sense of dying. He was quite conscious, called out, and was carried indoors and laid on a couch. Widespread clonic jerking of limbs and trunk then began and continued for about an hour. He was then admitted to a hospital, 
where coronary thrombosis was suspected, but an E.C.G. was normal. From that time to the present, a period of 10 years, he had suffered in the way described in the following notes. He gives a very clear account of his attacks. They occur only in his sleep or, more frequently, at the moment of dozing off to sleep. Naturally, therefore, they happen in bed at night, but may also occur if he dozes during the day. He describes three separate varieties of attack.

In the most severe he will be suddenly wakened from sleep or roused from the twilight state by a "jump ", seeming to start in the left precordial region, but involving all limbs and trunk and violent enough to twist him in bed. At the onset there is an involuntary contraction of the respiratory muscles, either in inspiration or expiration so that, as he says, sometimes the wind is forced out of him, sometimes forced into him. With this there is a quite involuntary cry. There is no loss of consciousness, and the whole eposide is over almost immediately. If he then keeps his eyes open and stays awake, so long as he does this he is free, but as soon as he closes them and begins to drowse off he has another attack. Thus they may continue for three or four times, or 60 or 70 times, so that he may not be able to get to sleep until three or four in the morning. In the second variety of attack the jerking is more circumscribed, being confined to the neck and perhaps the shoulder muscles. In the third, the spasm involves only the respiratory muscles, with consequent inspiratory or expiratory snort or gasp.

He declared himself well in every other way. He had resigned himself to his trouble, of which he was somewhat ashamed as he had been told it was neurotic, and sought the consultation with me while on holiday in this country from Australia. Travelling by train or motor coach was one of his bugbears as he had to keep a strict watch on himself to prevent dozing. On several occasions he had dropped off and had an attack which created a public disturbance. There was some doubt whether attacks occurred during sleep. His wife thought they did because after a jerk he would sometimes say, "Where am I ?", but the patient himself believed that he was never quite asleep when it seized him. There may, therefore, have been some disturbance of consciousness. An E.E.G. had been done two years before I saw him and was reported normal. There were no abnormal physical signs. As a result of taking sodium amytal, gr. 6, and " dexedrine", $10 \mathrm{mg}$., before going to bed he was improved, having occasional nights of complete freedom from the jerks but when he had a bad night it was as bad as ever.

Of considerable interest in this case is the attack at the age of 46, taking the form of a sudden collapse with angor animi, the type of seizure first described by Gowers (1907) as vaso-vagal and believed by Wilson (1928) and others, including myself (1950), to be an epileptic variant. It is to be noted that this attack had been preceded for several years by nocturnal jerks, was immediately followed by a brief myoclonic status, and thereafter by persistent nocturnal myoclonus. The following case is another in which there is a history of head injury and this a severe one.

Case 3.-This man, now aged 58 and a postman doing his work, first came under my care at hospital 20 years ago complaining of uncontrollable movements of the limbs at night, and his symptoms have continued with little change.

The family history in this case is perhaps relevant. The patient states that a brother at the age of about 21 suddenly developed what appears to have been a status epilepticus, was admitted to hospital, continued to have fits and died four or five weeks later, and was found at necropsy to have "a clot of blood in his head".

The patient himself sustained a gun-shot wound of the vertex in 1918. There was no loss of consciousness, but immediate paralysis of all four limbs. There was obviously a compound fracture of the vertex for operation was performed with removal of bone, leaving a cranial defect which was subsequently closed by means of a celluloid plate. At the end of six weeks he had recovered power in the arms and at the end of six months power in the legs had returned. They never in his own opinion became as strong as before, though he was quite able to do his work as a postman. Ever since his wound he has been liable to headaches, intermittent, worse in heavy weather or heat, at times severe like a drilling through the vertex with throbbing in the temples. Occasionally on bending forwards he had a sensation as if the inside of his head were falling forwards "like something in a basin going to spill. If I don't get up quickly I feel that something is going to spill ".

In 1930 his present symptoms began and have not altered. About every three weeks he had a bad spell for three or four nights in succession. Just as he is going off to sleep he has a sudden feeling as if particles all over his body are being drawn together by a magnet, leading in a flash to a violent jerk of all his limbs, the left more severely than the right : he cries out with it involuntarily but does not lose his senses. This may be immediately repeated. After a short interval there is recurrence, and so on for half an hour up to two hours, without any dozing in the intervals. He will then get up and take a dose of phenobarbitone ( 1 or 2 grains) and after this probably get off to sleep. Sometimes he may be wakened in the night by a jerk and when this happens the jerks are likely to recur for half an hour, or sometimes for the rest of the night. During a bad spell he is also liable to the jerks if he relaxes during the day and may be seized when sitting back in a comfortable seat in a cinema or theatre. During such a period he avoids sitting in an armchair at home. He has never had an attack in which he loses his senses.

When this patient first presented himself in 1932 no abnormal signs were found except impairment of postural sense in the toes, both right and left, and a left extensor plantar response. An E.E.G. in 1949 (Dr. Parsonage) was normal, and was again reported normal in 1950 (Dr. Kamal). 
The story of this man's gun-shot wound is that of an injury of the superior longitudinal sinus with slight residual damage to the brain. The preponderance of the myoclonus in the left limbs, corresponding as it does with the left extensor plantar response, is surely significant, but whether in this case the nocturnal myoclonus is to be attributed entirely to the brain injury is an open question. The significance of the family history is uncertain. It may be that an inherited epileptic liability has been disclosed and its form influenced by the injury. Nevertheless the history of a past gun-shot wound of the head in Case 1 has to be remembered in connexion with the present case in support of the view that nocturnal myoclonus may sometimes be a late effect of brain injury.

In the cases so far described the myoclonus has generally occurred under particular conditions, at the moment of dropping off to sleep or in a state of unusual mental and physical relaxation, though in the patients subject to myoclonus under these conditions it has on some occasions occurred during the night either in sleep or while awake. In this respect nocturnal myoclonus differs from that variety of myoclonic epilepsy in which seizures may be provoked by external stimuli, visual, auditory, tactile, or proprioceptive. The next case provides a link between these two conditions and is also of interest on account of the family history.

Case 4. - This man, aged 58, a plasterer, came under my care at hospital five years ago with the complaint of twitching of the legs. He gave the following account of his own complaint in other members of the family, in which there was no history of epilepsy.

His father, who died at the age of 67, used to complain of twitching legs, and the patient remembers seeing the twitching and recognizing it as the same as his own. This symptom he believes his father had had all his life. His mother was unaffected and was said to have died of old age. The patient gave the following account of his siblings.

Female, 66, affected in exactly the same way as the patient.

Male, 64, slightly affected : enough to complain.

Female, 60, affected in the same degree as the patient. Male, 54 , slightly affected.

Female, 47, he has not seen her for many years and does not know whether she is affected or not.

Female, 40, he has not seen her for some years, but knows that she was affected. She has now for several years been an invalid, leading a wheel-chair existence.

His past history included a fracture of the right humerus as a child and several operations for renal calculus, leading to a nephrectomy at the age of 52 .

At the age of 20 he first noticed involuntary twitching of the toes and calves in one or both legs, at first in the left only - mostly when resting-if he sat down to read a book, or went to the pictures, or in bed. This liability continued, and the twitchings became stronger and had now become a nuisance because they prevented sleep. He could seldom get to sleep before 2 or 3 a.m., though once he got to sleep the twitching would never wake him. If at work he has a slack half hour and settles down for a doze just as he is going off a twitch will occur. If then he gets up and goes for a walk it will cease. At night he frequently gets up and walks round the house in order to get off to sleep. He has also found that cutting a corn on his foot will cause a twitch if it unexpectedly hurts.

On examination he was an intelligent, cooperative man with no abnormal physical signs. The following observation was made upon the effects of peripheral stimulation. On scratching the sole there is an immediate, brisk, brief contraction of the whole quadriceps. With stronger stimulation the contraction is more prolonged, but never lasts more than a second. Immediate repetition of the stimulus causes repetition of the response. No other muscles are involved except for normal plantar flexion of the toes as a reflex. With very strong stimulation contraction occurs in both quadriceps at once. This spread occurs more easily from left to right. Spontaneous muscle contraction was also observed at rest in bed in a state of sleepy relaxation-brief volleys of clonic contraction of the quadriceps, the left more than the right, four or five shocks in succession.

The symptoms in this case are like those in the cases already described, differing, however, in the much more limited extent of the myoclonus, the continuous nature of the liability and the early age of onset. The additional feature of reflex myoclonus is important, and the family history of the complaint is that of a dominant inheritance.

In the next case there can be little doubt of the association of nocturnal myoclonus with frank epilepsy.

Case 5.-This man, aged 40 , is a crane driver at full work and presented himself in June, 1952, complaining of "jumps" preventing sleep. There is no family history of epilepsy or myoclonus. $\mathrm{He}$ is married with two children and has had no illness except pneumonia at 26 and a fracture of the right ankle at 28 . He has always been liable to faint easily at the sight of blood or other disagreeable spectacle and is apt to feel faint if he stands for any length of time. He then sits down with his head between his knees and things go black for a minute or two, but he remains conscious. Apart from this, during the past 20 years he has had occasional, causeless attacks of unconsciousness, sometimes when sitting, preceded by a brief feeling of giddiness. As a rule he recovers his senses in a few minutes, but on two occasions there has been amnesia for three-quarters of an hour. He has never bitten his tongue or passed urine in an attack. 
Towards the end of 1951 he began to suffer from jumps at the moment of going off to sleep. At first these were confined to the left leg-a momentary, involuntary jerk, after which he would doze off, to be disturbed by recurrence at intervals of a minute or two for 10 minutes. He then began to get more prolonged attacks, lasting one to three hours, keeping him awake, and as time passed the jerking on occasions would involve all his limbs, the trunk, and head. The face was never affected. $\mathrm{He}$ also began to have these jumps in the day whenever he lay and relaxed, for example, on a Saturday afternoon. His wife states that they also occurred in his sleep. He was able to stop the jumps by getting up and moving about and would often do so at night in the attempt to get off to sleep, but in the earlier part of the night whenever he returned to bed and relaxed they would start again. Several episodes were seen while he was in bed in the ward, but none were effectively recorded. On one occasion he dozed when I was discussing his case, and there was a momentary jerk of the lower limbs, enough to disturb the bed clothes. There were no abnormal physical signs.

An E.E.G. was recorded by Dr. Pond after drowsiness had been induced by 3 grains of " seconal ". It was marked by runs of slow activity in the posterior regions, some of the waves being rather sharp, but symmetrical. The frontal regions remained relatively fast and did not show any increased amount of fast activity as should have happened from the barbiturate. There were several episodes of jerking of the left leg. On one occasion this appeared to be associated with a slight asymmetry in the central regions, but usually quite symmetrical arousal patterns were obtained with a burst of fast rhythm that seemed to be the patient's alpha rhythm. No epileptic activity was seen at any time. The effects of photic stimulation were normal. Dr. Pond's opinion was that this E.E.G. was in favour of some diffuse or basal lesion.

He was given various barbiturates at bed-time with little or no effect. Finally on November 26 two tablets of " tridione" at bed-time were prescribed. A week later he reported better nights than he had had for many months. He had only had the jumps on three nights out of seven and then not for more than two or three minutes and involving a single limb-right lower, left lower, and right upper, on separate occasions. Seen again a month later, he reported satisfactory progress, the jumps occurring seldom and continuing for no longer than a few minutes. The consequent gain in sleep had restored him to his feeling of normal health.

The affinity between this case and the others is clear, and there can be little doubt of the occurrence in the past of epileptic seizures. The excessive liability to fainting cannot be passed over as a coincidence for its occasional association with epilepsy is undoubted. The response of the nocturnal myoclonus to tridione in this case has been striking. This drug was tried in Case 3 with little effect, but was not given in the other cases.

\section{Discussion}

Involuntary, shock-like muscular contractions, limited or widespread, without loss of consciousness, have been recognized as a common accompaniment of idiopathic epilepsy for more than a century. Russell Reynolds (1861) in a series of 57 cases found that $81 \%$ had clonic symptoms of this kind between fits, and Muskens (1928) from his observations upon more than 2,000 cases endorsed this statement, adding that in most cases the shocks had been present for many years before the first fit. As these and other writers have observed, it is in the first hour after waking in the morning that the epileptic is most liable to these myoclonic jerks, and in those whose epilepsy is periodic, the myoclonus is apt to recur at this time for several mornings in succession before a longer series of the jumps (as they are usually called by the patient) leads up to a generalized convulsion with loss of consciousness.

It is also well known that in some epileptics myoclonus may be provoked by sensory stimuli, especially flicker-photic stimulation. Noise, startle, unexpected touch, and passive stretching of muscle are among the other effective stimuli. Apart from this the initiation of voluntary movement in some cases will frequently provoke myoclonus if the patient is in a bad phase. Wohlfart and Hook (1951) have emphasized this point in a clinical analysis of 10 cases of myoclonic epilepsy and describe the symptom as action myoclonia. In only two of their cases did myoclonus occur when the patient was at rest and completely relaxed. It appears to be the general rule (Van Bogaert, Radermecker, and Titeca, 1950) that the myoclonus of epileptics ceases during sleep, but to this rule there are exceptions. Pierce Clark (1912) studied one such case and found that the myoclonus never occurred when the patient was in a state of deep and quiet sleep, but whenever he turned over in bed or greatly shifted the position of his body the contractions were sure to occur with great violence and woke him. This then was an action myoclonus occurring during sleep.

Less attention has been paid to the occurrence of myoclonus in known epileptics at the moment of going off to sleep, but Muskens (1928) records a case which may be summarized as follows. A man aged 28 had suffered from myoclonic shocks characterized by extension forward of the arms for six years. These shocks came if he lay down, particularly if he dropped asleep. He therefore avoided the recumbent position as far as possible. Soon after the shocks the patient began to have fits, from two to eight in a month. Muskens further 
says, in discussing the fact that myoclonic convulsions of this kind are often not recognized as epileptic, that they certainly occur in sleep and frequently awake the patient. " Very frequently such a convulsion occurs just at the moment of dozing off and awakes the patient." He records also that in his experiments with cats rendered epileptic by injections of mono-bromide of camphor he had frequently observed the occurrence of myoclonus at the moment of falling asleep as well as awakening.

Hodskins and Yakovlev (1930) observe that some epileptics have myoclonus during the hypnogogal period preceding sleep and believe that the accompanying alteration of postural tone is the precipitating cause. Van Bogaert and others (1950) also note the facilitation of myoclonus by going to sleep in some cases of epilepsy with myoclonus. Other forms of epileptic seizure may also occur at this particular moment. A woman aged 40 at present under my care has had major and minor seizures since she was 12 . For many years they have occurred in spells with intervals of freedom for several weeks. The minor attacks take the form of a typical dreamy state in which her thoughts become fixed upon something she is trying to remember. On the very brink of reminiscence she loses her senses for a moment. In the major attacks the "thought" goes on to a generalized convulsion. The first intimation of a bad spell is that just as she is going off to sleep the thought comes and she wakes with a start.

This may be repeated several times before she finally goes to sleep. This recurs on the following night, and then she may be wakened from sleep once or twice in the night, coming suddenly to her senses with the knowledge that the thought has been there. Subsequently she has minor attacks in the day, leading up to one or more major attacks, almost all nocturnal. Following this the sequence is reversed. After a day or two she is having the thoughts only when dropping off to sleep, and when this ceases she knows that she will have another interval of freedom.

In the light of these observations it seems probable that the syndrome of nocturnal myoclonus, as described in this paper, is an epileptic variant, though only one patient, Case 5 , had actual epileptic attacks. Case 4 differs from the others in many respects, yet the reflex myoclonus suggests an epileptic character, and though the jerks were confined to the legs the conditions under which they appeared were the same as in the other cases.

For any connexion between the cases described in this paper and the common nocturnal jerks I can find no evidence apart from the impression already recorded that a history of excessive nocturnal jerks is sometimes to be obtained from patients with idiopathic epilepsy of late onset, but the question of a relationship between nocturnal jerks and nocturnal myoclonus, and the possibility of a familial incidence has clearly occurred to others. Muskens (1928), for example, states that cases are met with where in a family there exists a greater tendency to myoclonic discharges than is quite normal and epilepsy suddenly appears. Van Bogaert and others (1950) state : "Plenty of people with no clinical or electrical evidence of epilepsy may present myoclonic twitches in one or other limb, more especially at the moment of going off to sleep. However an E.E.G. has never to our knowledge been obtained during these twitches." But, Perlstein, Gibbs, and Gibbs (1947), in discussion replying to the question whether the type of sudden jerk that is very common in normal persons when falling asleep is associated with any abnormality of the electrical sleep record, stated that they had studied this in more than 50 normal persons and had observed such jerking many times without associated E.E.G. abnormality. This corresponds with the observation of Dr. Pond in Case 5. Pond (1953) suggests that in this case theo absence of any specific discharges in the E.E.G. at the moment of the jerk may be, first, that the cortical spike component is very small, so that it旅 might be missed without special electrode positions, and, second, that the discharge may be entirely at some lower centres without a cerebral componentRoger and Gastaut (1951) in an interesting discussion of the myoclonic threshold in normal man, when attacks are induced by the combination of cardiazol injection and photic stimulation, consider the variable factors and insist upon the importance of eye closure in lowering the threshold. This may be significant and suggests that it might be of value to observe whether the muscular contraction occurs at the moment of eye closure in dozing, both in nocturnal jerks and nocturnal myoclonus.

\section{Summary}

Five cases are recorded in which the patient's complaint was that of involuntary jerking or twitching of the limbs or body occurring at the moment of falling asleep, during sleep, or in a state of mental and physical relaxation during the day. In each case the symptoms interfered seriously with sleep.

Evidence is presented from these cases and from other sources for supposing that this syndrome of nocturnal myoclonus is an epileptic variant which may be familial.

The relationship of nocturnal myoclonus to the nocturnal jerks not infrequently experienced by 
normal persons at the moment of falling asleep is briefly discussed.

\section{REFERENCES}

Clark, L Pierce (1912). J. Amer. med. Ass., 58, 1666.

Gowers, W. R. (1907). The Border-Land of Epilspsy. Churchill,

London.
Hodskins, M. B., and Yakovlev, P. I. 1930). Amer. J. Psychiat., 86, (n.s. 9). 827 .
Muskens, L. J. J. (1928). Epilepsy. Baillière, Tindall and Cox, London.

Perlstein, M. A., Gibbs, E. L., and Gibbs, F. A. (1947). Res. Publ. Ass, nerv, ment. Dis., 26, 366.

Pond, D. A. (1953). Personal communication.

Pond, D. A. (1953) il (1861). Epilepsy. Churchill, l.ondon.

Roynor, A., and Gastaut, H. (1951). Rev. Neurol., P(ris, 84, 612.

Symonds, C. (1950). Proc. roy. Soc. Med., 43, 507.

Van Bogaert, L., Radermecker, J., and Titeca, J. (1950). Folia psychiat, Amst., 53, 650.

Wilo psyl, A (1928) J, Neurol. Psychopath. 8, 223.

Wohlfart, G., and Hook, O. (1951). Acta psychiat. Kbh., 26, 219. 